\title{
Simulação da partida de reator nuclear de pesquisa utilizando instrumentos virtuais
}

\author{
Startup simulation of nuclear research reactor using virtual \\ instruments
}

\author{
Amir Zacarias Mesquita \\ amir@cdtn.br \\ Centro de Desenvolvimento \\ da Tecnologia Nuclear/ \\ Comissão Nacional de \\ Energia Nuclear
}

\section{Antônio Juscelino Pinto ajp@cdtn.br \\ Centro de Desenvolvimento da Tecnologia Nuclear/ Comissão Nacional de Energia Nuclear}

\section{Aldo Márcio Fonseca Lage aldo@cdtn.br \\ Centro de Desenvolvimento da Tecnologia Nuclear/ Comissão Nacional de Energia Nuclear}

\section{Eldrick D`Martins}

eldrick@oi.com.br Centro de Desenvolvimento da Tecnologia Nuclear/ Comissão Nacional de Energia Nuclear

\author{
Wallen Ferreira de Souza \\ wallenfds@yahoo.com.br \\ Centro de Desenvolvimento \\ da Tecnologia Nuclear/ \\ Comissão Nacional de \\ Energia Nuclear
}

\begin{abstract}
Resumo
Este artigo descreve o sistema digital desenvolvido para simulação da evolução temporal das variáveis relacionadas ao fluxo neutrônico de reator nuclear de pesquisa TRIGA IPR-R1, do Centro de Desenvolvimento da Tecnologia Nuclear (CDTN). Foi utilizado o software LabVIEW ${ }^{\circledR}$, que emprega o moderno conceito de instrumentos virtuais por meio de processador eletrônico e interface visual em monitor de vídeo. O objetivo é auxiliar no treinamento dos estudantes de engenharia nuclear e operadores de reatores, permitindo observar e analisar o comportamento e a tendência de alguns dos processos que acontecem em um reator nuclear. Deste modo, os parâmetros de operação do reator podem ser simulados e seus relacionamentos visualizados, auxiliando no entendimento de como essas variáveis estão interligadas e se comportam. O sistema possibilitará o estudo de parâmetros que afetam a operação do reator sem a necessidade de usar a instalação. O sistema, além de simular, poderá também monitorar o comportamento de todas as variáveis operacionais da instalação.
\end{abstract}

Palavras-chave: Parâmetros neutrônicos. LabVIEW ${ }^{\circledR}$. Instrumentos virtuais. Simulação digital. Reator nuclear TRIGA.

\begin{abstract}
This paper describes the digital system developed to simulate the temporal evolution of variables related to the neutron flux of the IPR-R1 TRIGA nuclear research reactor at Nuclear Technology Development Center (CDTN). The system was developed using the LabVIEW ${ }^{\circledR}$ software that uses the modern concept of virtual instruments by electronic processor and visual interface in video monitor. The goal is to help the nuclear engineering students and reactor operators training, allowing to observe and analyze the behavior and the trend of some processes occurring in a nuclear reactor. Thus, the reactor operation parameters can be simulated and their relations can be visualized, helping in understanding how these variables are interconnected and behave. The system will enable the study of factors affecting the operation of the reactor without the need to use the facility. The system besides simulating can also monitor the behavior of all operational parameters of the installation.
\end{abstract}

Keywords: Neutronic parameters. LabVIEW ${ }^{\circledR}$. Virtual instruments. Digital simulation. TRIGA nuclear reactor.

\section{Introdução}

Além da produção de energia elétrica, o renascimento do uso e aplicação da energia nuclear vem ocorrendo em outros segmentos, como na medicina, para a produção de radiofármacos, na indústria e na irradiação de alimentos, demandando treinamento especializado de profissionais para desempenhar estas atividades em instalações radiativas e nucleares. Para que elas sejam realizadas com segurança, confiabilidade, qualidade e credibilidade, é essencial a utilização de instrumentação que atenda a alguns requisitos.

A maior vantagem ambiental da geração elétrica através de usinas nucleares é a não utilização de combustíveis fósseis, evitando o lançamento na atmosfera dos gases responsáveis pelo aumento do aquecimento global e outros produtos tóxicos. Usinas nucleares ocupam áreas relativamente pequenas, podem ser instaladas próximas aos centros consumidores 
e não dependem de fatores climáticos para o seu funcionamento. Além da manutenção dos reatores em operação, tem aumentado o número de reatores em construção e centenas estão em fase de projeto em todo o mundo. No Brasil a entrada em operação de Angra 3, em 2018, e a previsão da construção de mais quatro centrais de potência, além do projeto e construção do Reator Brasileiro Multipropósito (RBM), que será um reator de pesquisa com algumas características similares ao IPR-R1, exigirão pessoal qualificado em todos os setores da energia nuclear (CNEN, 2008).

O número de reatores de pesquisa que podem ser utilizados para estudos e formação na área nuclear no país ainda será pequeno diante desse cenário. Portanto, sistemas virtuais, nos quais sejam possíveis simulações e estudos de comportamento dos reatores, sejam de potência, sejam de pesquisa, são de essencial importância para suprir essas necessidades e apoiar esse avanço na área nuclear no país. Outra vantagem é a economia de recursos financeiros e humanos, uma vez que podem ser utilizados independentemente do reator.

O reator nuclear de pesquisa TRIGA IPR-R1, do Centro de Desenvolvimento da Tecnologia Nuclear (CDTN), em Belo Horizonte, começou a operar em 1960 e foi o segundo reator nuclear instalado no país. Até o momento, só existem dois reatores de pesquisa desse porte no Brasil. Dentre suas aplicações está o treinamento de profissionais que atuam na área nuclear. Inserido Um sistema para simulação da evolução do fluxo de nêutrons foi desenvolvido com o intuito de auxiliar no treinamento de operadores de reatores, permitindo estudar, observar e analisar o comportamento e a tendência de alguns dos processos que acontecem em um reator. Deste modo, os parâmetros de operação do reator podem ser simulados e seus relacionamentos visualizados sem a necessidade de colocar o reator em operação.

O sistema foi desenvolvido utilizando o software LabVIEW $^{\circledR}$ e aplicando o conceito de instrumentos virtuais (VI). Foram desenvolvidos diversos VIs com as curvas usadas no sistema, por exemplo, a equação da curva real, que relaciona a posição da barra de regulação com a reatividade inserida por ela no processo, e a equação da curva de Inhour. Os VIs permitem ao usuário visualizar e analisar como se comporta a evolução do fluxo de nêutrons, em função do tempo de operação, do período e da potência inicial, tanto para o canal linear, quanto para o canal logarítmico, e também fazer a análise da relação entre o reposicionamento da barra de regulação do reator e a reatividade real inserida no reator pelo reposicionamento da barra.

As simulações permitem também avaliar os efeitos de valores extremos nas diversas variáveis e sem qualquer risco. Com relação à instrumentação, o documento da IAEA (2002) destaca o conceito de controle automático, principalmente para desligamento: "a reação humana é muito mais lenta do que a dos instrumentos e a atenção humana não consegue ficar constantemente focada em todos os parâmetros de segurança". Assim, é recomendada explicitamente a existência de uma interface amigável para o controle de reatores nucleares, uma vez que "a monitoração e controle dos sistemas importantes para a segurança envolvem uma combinação de: funções de medidas e controle automáticos e monitoração e controle por operadores humanos". O conhecimento de todas as variáveis inerentes ao processo e suas implicações são de extrema importância para a operação segura dos reatores (IAEA, 2008).

\section{0 reator de pesquisa TRIGA IPR-R1}

O reator nuclear de pesquisa TRIGA IPR-R1 foi adquirido da General Atomics pelo governo do estado de Minas Gerais, em 1960, por meio do programa do governo americano "Átomos para a Paz". Foi instalado no antigo Instituto de Pesquisas Radioativas (IPR), atual Centro de Desenvolvimento da Tecnologia Nuclear (CDTN), da Comissão Nacional de Energia Nuclear (CNEN). Apesar de sua idade, seu combustível está pouco "queimado" (cerca de 4\% em média), podendo operar ainda por vários anos na realização de pesquisas, na produção de radioisótopos e em treinamentos exclusivos desse tipo de instalação. A Figura 1 mostra uma foto do poço e uma foto do núcleo do TRIGA IPR-R1, nas quais se pode ver a radiação de Cerenkov quando o reator está crítico.

O reator IPR-R1 é um reator de pesquisas refrigerado à água leve desmineralizada e seu combustível é uma liga metálica de urânio com o moderador hidreto de zircônio (U-ZrH) contendo de $8 \%$ a $8,5 \%$ em peso de urânio enriquecido a $20 \%$ em ${ }^{235} \mathrm{U}$. O núcleo do reator TRIGA IPR-R1 forma um reticulado cilíndrico, onde estão atualmente instalados 63 elementos combustíveis-moderadores, sendo 59 elementos originais, revestidos com alumínio, e 4 elementos inseridos recentemente, com revestimento em aço inoxidável. 
Figura 1 - Reator nuclear de pesquisa TRIGA IPR-R1 vista do poço (a) e núcleo com o reator ligado (b).

(a)

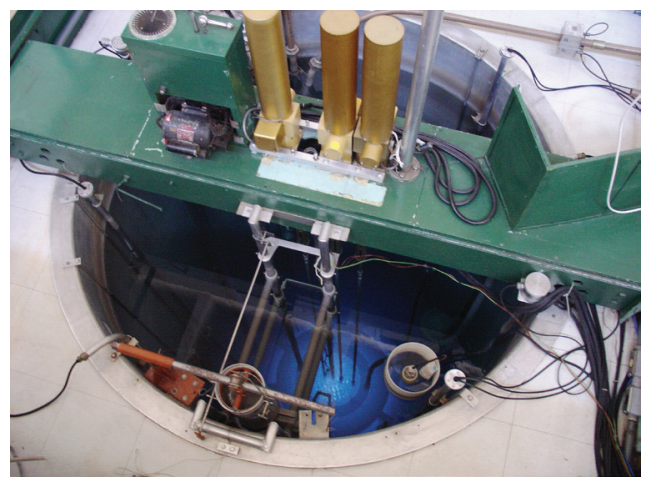

(b)

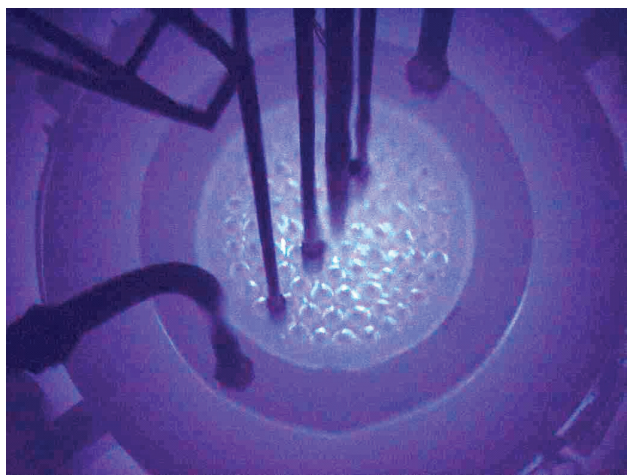

Fonte: Arquivo pessoal.

A potência máxima do reator IPR-R1 era de $30 \mathrm{~kW}$ térmicos inicialmente, mas posteriormente houve um acréscimo de elementos combustíveis ao núcleo, aumentando a potência para $100 \mathrm{~kW}$, a atual potência máxima licenciada. Foram realizadas modificações no núcleo em 2004 e acrescentaram-se novos elementos combustíveis, permitindo que a potência atinja níveis de $250 \mathrm{~kW}$ (MESQUITA, 2005).

Os reatores TRIGA (Training, Research, Isotopes, General Atomics) são os reatores de pesquisa mais amplamente utilizados. Conforme indica sua sigla, foram desenvolvidos, principalmente, para formação de pessoal e pesquisas. Esses reatores são caracterizados por sua segurança intrínseca devida principalmente a dois fatores relacionados à transferência de calor, são eles:

- O grande coeficiente negativo de temperatura/reatividade, isto significa que um aumento da potência leva a um consequente aumento da temperatura da mistura combustível-moderador, causando o aparecimento de uma reatividade negativa que amortece gradualmente a taxa de aumento de potência, e estaque tende a se estabilizar.

- Um sistema passivo de remoção de calor do núcleo, podendo operar em potências de até $500 \mathrm{~kW}$, em estado estacionário, com resfriamento apenas por circulação natural da água da piscina.

Portanto, os reatores TRIGA são bastante apropriados para formação de pessoal e pesquisas experimentais em termo-hidráulica e neutrônica. Eles estão, em sua maioria, instalados em universidades, hospitais, indústrias e centros de pesquisa de vários países.

\subsection{Controle da potência do reator TRIGA}

O controle da potência do reator TRIGA IPR-R1 é realizado pela movimentação axial de três barras de controle no núcleo em uma faixa de aproximadamente $35 \mathrm{~cm}$. As posições totalmente inseridas correspondem a um número arbitrário, igual a 150, e as posições totalmente retiradas correspondem ao número 900. As barras de controle são constituídas de carboneto de boro e se movimentam em tubos-guia de alumínio, perfurados e fixados na grade inferior do núcleo. A Figura 2 apresenta a seção longitudinal do reator TRIGA IPR-R1, onde podem ser vistas as barras de controle e as câmaras detectoras de nêutrons, dentre outros dispositivos. A Figura 3 apresenta o diagrama do núcleo (MESQUITA, 2005). As três barras de controle do IPR-R1 são assim denominadas:

- Barra de segurança: projetada para atuar nos casos de emergência, em que provoca um desligamento rápido do reator. Ela se encontra totalmente extraída durante a operação.

- Barra de controle (controle grosso): destinada a compensar grandes variações de reatividade, como as decorrentes de envenenamento (reatividade negativa) por produtos de fissão, queima de combustível (em longo prazo), por dispositivos experimentais, etc.

- Barra de regulação (controle fino): destinada a compensar pequenas variações de reatividade, como aquelas provocadas por pequenas variações de temperatura e pequenos envenenamentos por amostras introduzidas. 
Figura 2 - Seção longitudinal do reator TRIGA IPR-R1.

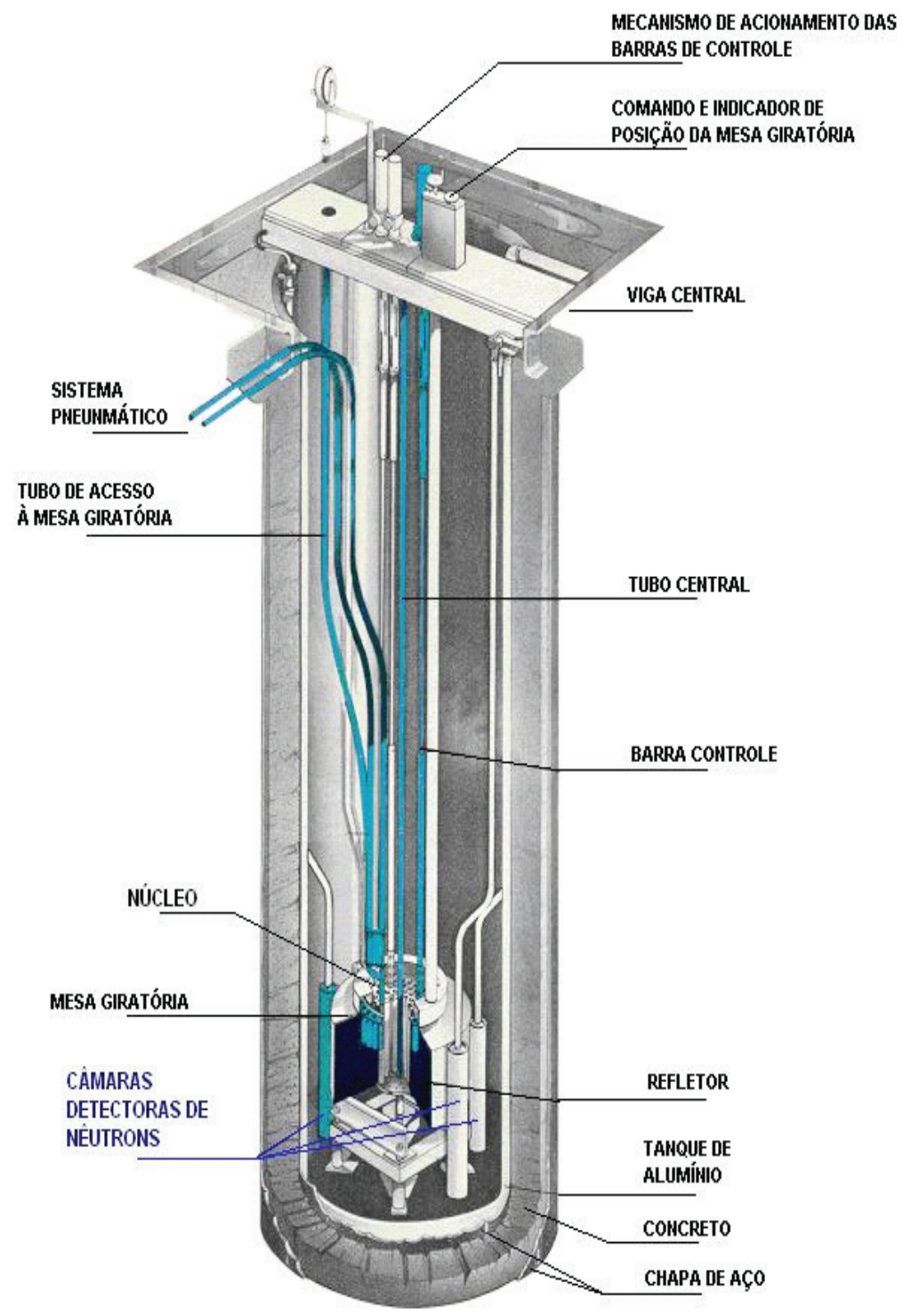

Fonte: Mesquita (2005).

As barras de controle do reator permitem ajustar o valor de K (fator de multiplicação de nêutrons) do sistema, tornando-o maior do que $1(>1)$ nas subidas de potência, igual a $1(=1)$ em potência estacionária e menor que $1(<1)$ nas reduções de potência, ou no desligamento. Essas alterações de $\mathrm{K}$ ocorrem essencialmente por variações da absorção de nêutrons no núcleo. Retirando-se as barras do núcleo, diminuem-se as absorções e o $\mathrm{K}$ aumenta; ao contrário, introduzindo-as no núcleo, aumentam-se as absorções e o K diminui. A eficiência de uma barra de controle, em termos de sua capacidade de alterar a reatividade do sistema, depende do valor do fluxo no local de inserção. De acordo com esse conceito, a b Barra de regulação deverá ter menor eficiência no controle da reatividade que as barras de controle e de segurança. Assim, como pode ser visto na Fig. 3, a barra de regulação (F16) desloca-se na periferia do núcleo (anel F), enquanto as barras de controle $(\mathrm{C} 1)$ e a de segurança $(\mathrm{C} 7)$ deslocam-se mais próximas ao centro (anel $\mathrm{C}$ ). 
Figura 3 - Configuração do núcleo do reator TRIGA IPR-R1.

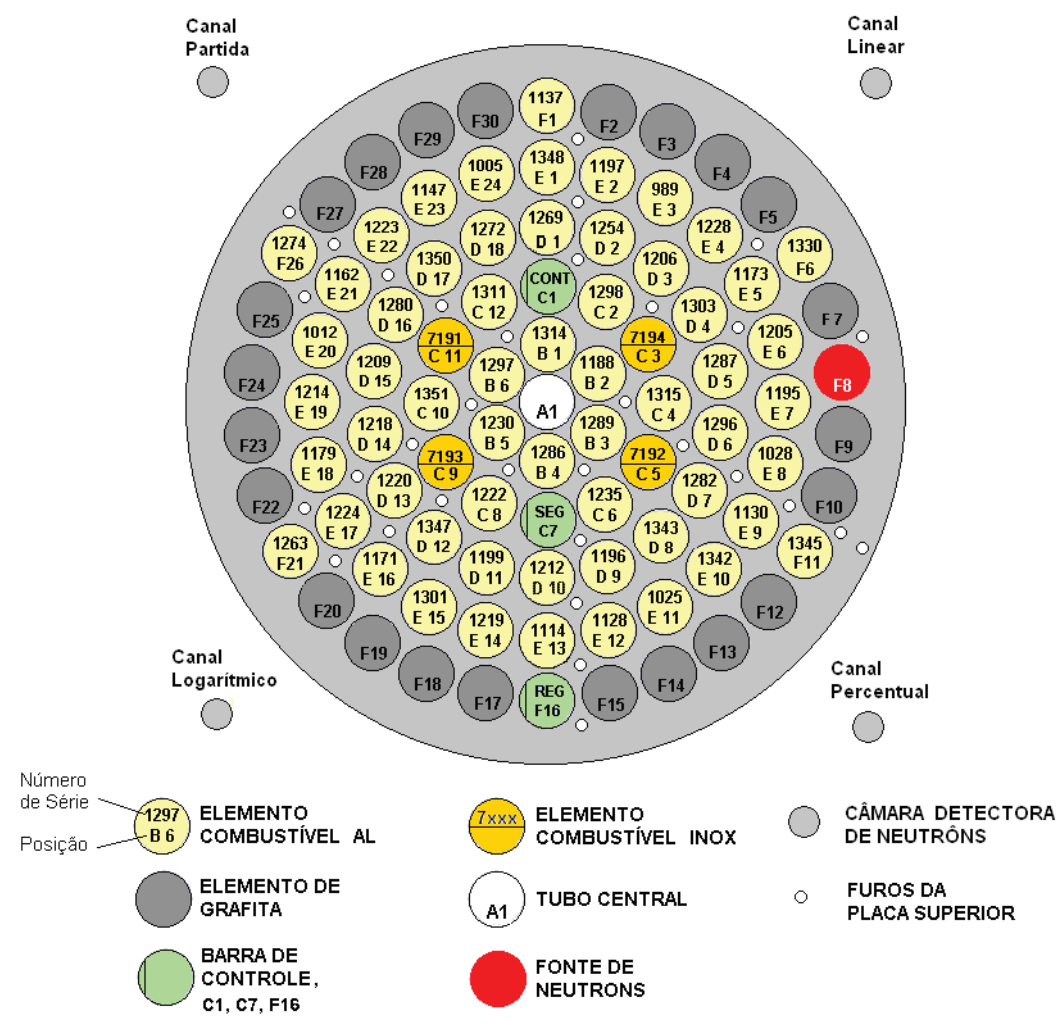

Fonte: Mesquita (2005).

\section{Metodologia}

O software LabVIEW ${ }^{\circledR}$ (Laboratory Virtual Instruments Engineering Workbench), versão 8.6, da National Instruments (2007), é uma poderosa ferramenta, sendo o programa mais utilizado atualmente, tanto para aplicações industriais como em controle de reatores. Além de poder trabalhar com os modernos sistemas operacionais, como Windows XP e Vista (PEDERSON, 2005; TIGLIOLE, 2008), o LabVIEW ${ }^{\circledR}$ oferece controle direto do hardware das placas de aquisição de dados. Ele é programado com uma série de ícones gráficos, os quais são conectados com "fios". A combinação das placas DAQ adequadas e o software LabVIEW ${ }^{\circledR}$ possibilitam a criação de instrumentos virtuais (IV). Um VI desempenha funções semelhantes aos instrumentos tradicionais, mas é programado via software, com flexibilidade e a vantagem de registro dos dados que estão sendo medidos.

Os ícones no LabVIEW ${ }^{\circledR}$ representam controles e funções disponíveis nos menus do software, chamado de programação visual. A interface do usuário consiste de duas partes: o painel frontal e o diagrama de blocos, semelhante aos instrumentos tradicionais, que possuem um painel frontal e a placa de circuito impresso onde reside o circuito eletrônico. No painel frontal do LabVIEW $^{\circledR}$, podem-se criar os botões de controle, chaves, leds e displays gráficos para apresentar os dados enquanto que, no diagrama de blocos, residem os blocos que, adequadamente interligados, constituem o circuito eletrônico. Um VI também possui funções matemáticas, podendo calcular a média, e o desvio padrão e apresentá-los graficamente. Possui alarmes que podem ser configurados para indicar ao usuário o status do processo e realizar o armazenamento dos dados em arquivos para análise posterior. O LabVIEW ${ }^{\circledR}$ pode ser usado para realizar a simulação de sistemas, porque ele contém uma infinidade de recursos, como filtros, processamento de sinal digital e funções estatísticas. 


\subsection{Simulação dos parâmetros usando o software LabVIEW ${ }^{\circledR}$}

Uma interface homem-máquina foi desenvolvida levando em consideração a Equação do Inhour, que relaciona reatividade $(\rho)$ com periodo $(T)$, isto é, o afastamento da condição de criticalidade com a velocidade do afastamento. A simulação somente é válida para reatores que estão críticos, ou seja, com potência estacionária, em que a reatividade $(\rho)=0$ e o fator de multiplicação de nêutrons $(\mathrm{K})=1$. (CDTN/CNEN1997)

Este trabalho também leva em consideração que a barra de segurança se encontra totalmente extraída do núcleo reator, já que ela possui reatividade suficiente para produzir o scram (desligamento) e que a barra de controle está fixa na posição 500, por exemplo, condição que permite a partida do reator. Finalmente, este estudo também considera um limite máximo de potência de $1000 \mathrm{~W}$, pois, após este nível, começa a aparecer no processo o efeito de temperatura/reatividade, o qual interfere no crescimento neutrônico.

\subsection{Gráfico da reatividade em função da posição de barra de regulação}

Considerando que cada barra de controle do reator possui uma determinada reatividade ( $\rho$ ) total negativa e que cada segmento de barra, ao ser introduzida, ou retirada do reator, insere-se ou retira-se um $\Delta \rho$ de reatividade negativa, adicionou-se no programa a curva real, que relaciona a reatividade inserida no núcleo, pela barra de regulação, em função de sua posição. Os valores foram obtidos por meio dos experimentos realizados no reator IPR-1 por Mesquita e Souza (2014). Neste gráfico foram implementados cursores e indicadores que informam ao usuário a reatividade inserida no reator pela barra de regulação em função do seu posicionamento, como pode ser visto na Figura 4.

Figura 4 - Reatividade em função da posição da barra de regulação.

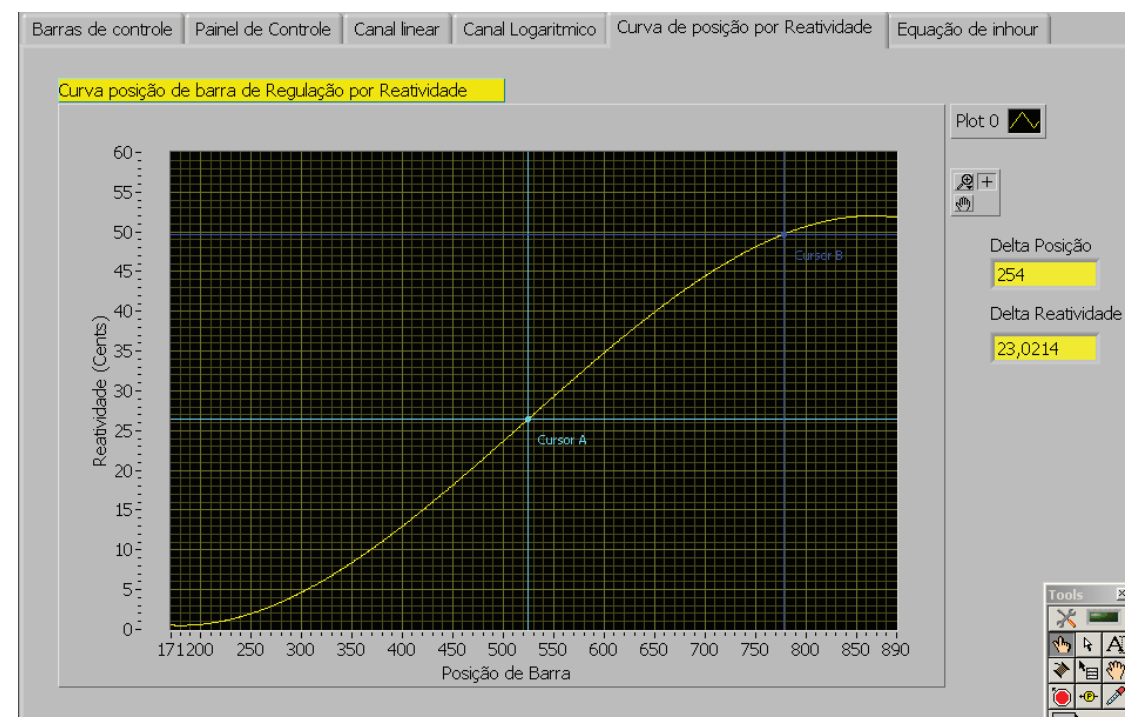

Fonte: Pinto (2011).

\subsection{Gráfico da Equação do Inhour}

A equação que relaciona a reatividade $(\rho)$ com o período (T) (intervalo de tempo no qual a quantidade de nêutrons cresce de um valor igual ao número $e=2,72$ ), é a chamada Equação do Inhour, dada por:

$$
\rho=\frac{\ell}{T(1+\delta k)}+\sum_{i=1}^{6} \frac{\beta_{i}}{1+\lambda_{i} T}
$$

Ela leva em consideração o tempo médio $\ell$ que separa duas gerações consecutivas de nêutrons. No caso do reator IPR-R1, é de $100 \mu \mathrm{s}$, a variação do fator de multiplicação ( $\delta \mathrm{k})$, e os seis grupos efetivos de nêutrons atrasados, sendo que cada grupo é caracterizado pela sua constante de desintegração $\lambda_{i}$ do precursor e pela proporção $\beta_{i}$ de nêutrons em relação ao número de nêutrons de fissão. Os dados relativos aos nêutrons atrasados para fissão térmica do ${ }^{235} \mathrm{U}$, utilizados no gráfico de Inhour, podem ser encontrados em CDTN/CNEN, 1997. 
Desta forma e de posse da reatividade inserida no reator, agora conhecida, o usuário consulta a curva de Inhour e, por meio dos cursores e indicadores implementados no gráfico, verifica para essa reatividade qual é o período do crescimento neutrônico, conforme mostrado na Figura 5.

Figura 5 - Equação do Inhour.

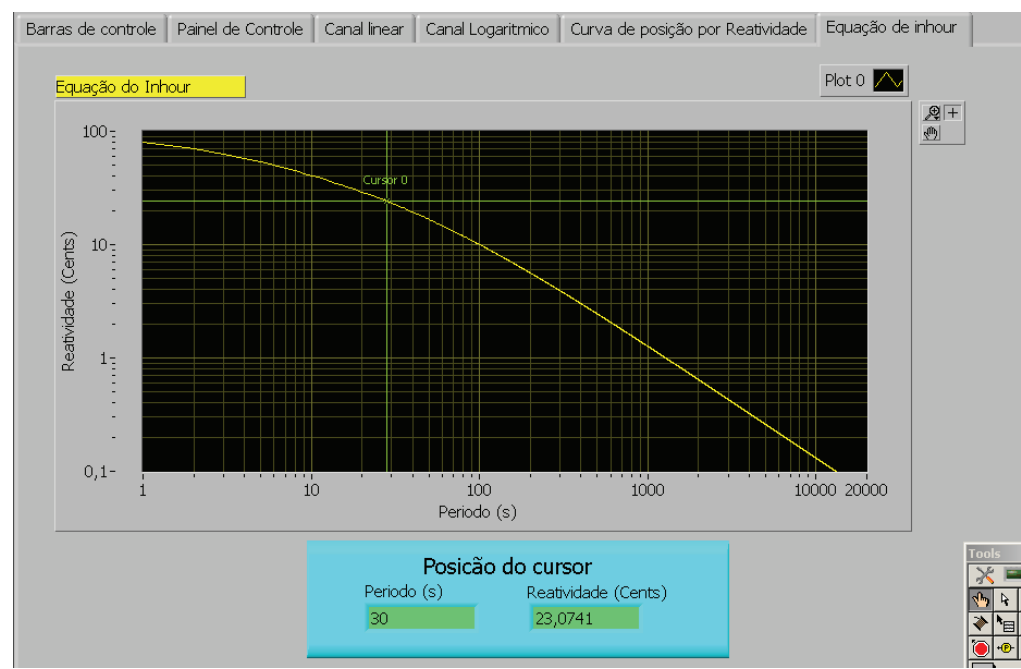

Fonte: Pinto (2011).

\subsection{Gráficos dos canais linear e logarítmico}

Agora, com o período conhecido, na tela "Painel de controle", o usuário escolhe as opções de apresentação dos gráficos, a velocidade de simulação e insere o período, como mostrado da Fig. 6. As evoluções do crescimento neutrônico dos canais linear e çogarítmico são mostradas em suas respectivas telas no LabVIEW. As curvas do crescimento neutrônico mostradas são obtidas pela equação:

$$
N(t)=N_{o} * e^{t / T}
$$

Onde $\mathrm{N}(\mathrm{t})$ é o fluxo de nêutrons, $\mathrm{N}_{0}$ é o fluxo de nêutrons inicial, $\mathrm{t}$ é o tempo em segundos e $\mathrm{T}$ o valor do período em segundos. Assim, o usuário pode analisar e estudar o crescimento neutrônico nos canais linear e logarítmico, como mostrado nas Figs. 7 e 8 respectivamente.

Figura 6 - Painel de controle.

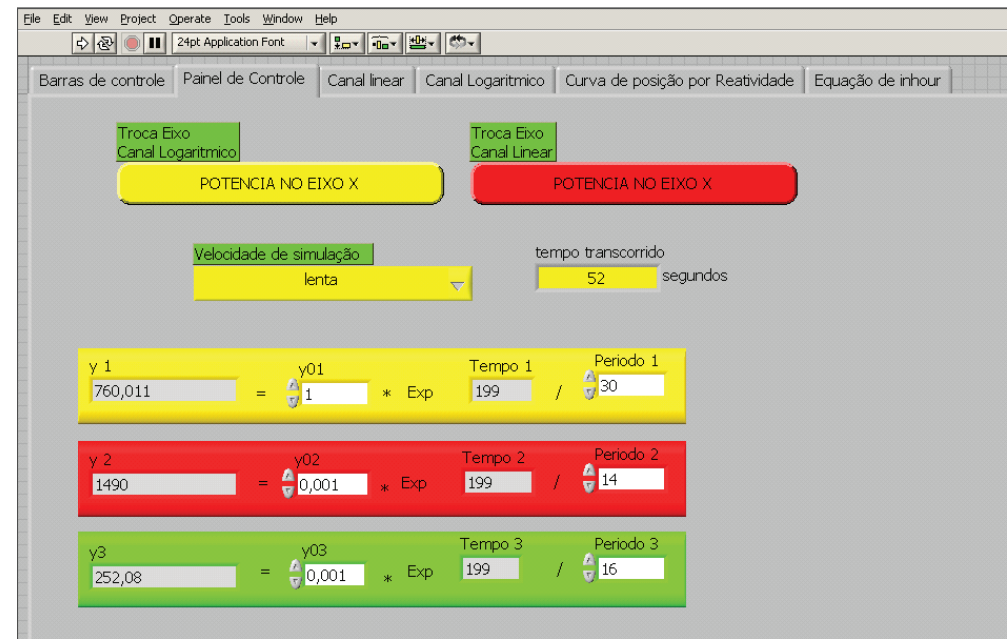

Fonte: Pinto (2011). 
Figura 7 - Comportamento do canal linear.

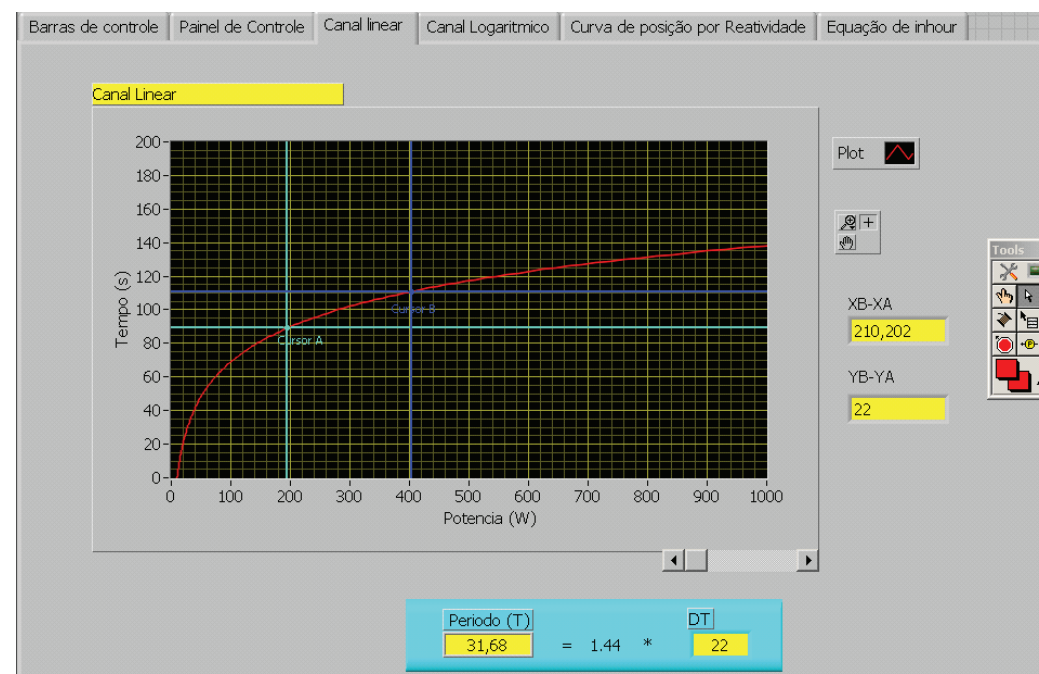

Fonte: Pinto (2011).

Figura 8 - Comportamento do canal logarítmico.

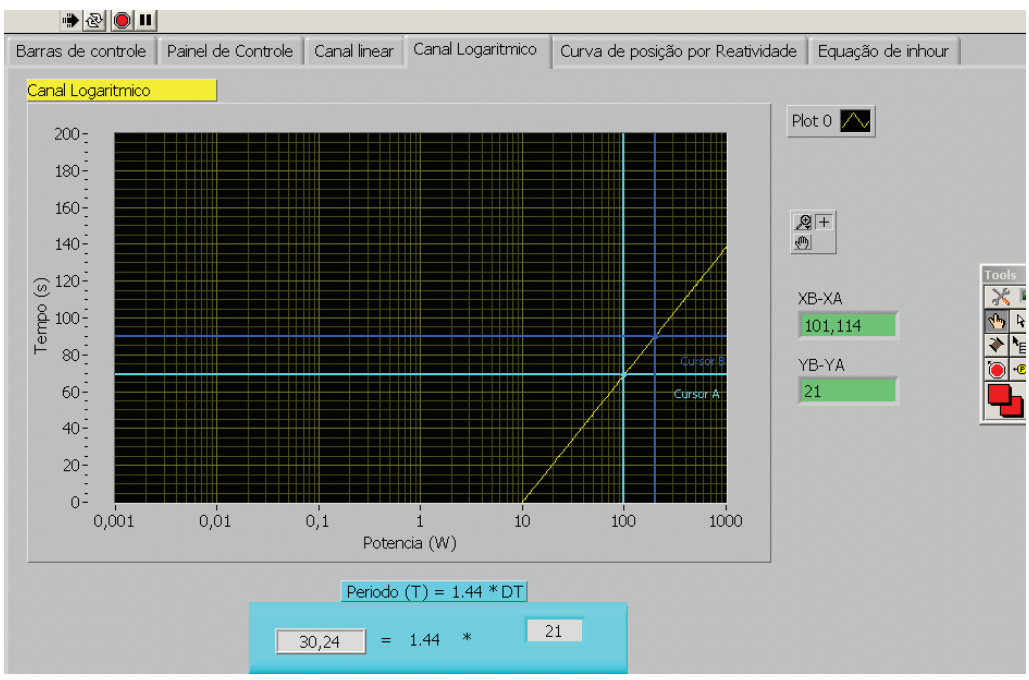

Fonte: Pinto (2011).

Observa-se ainda que, nesses dois gráficos, o usuário pode obter, por meio dos cursores e indicadores implementados, o tempo de dobramento da potência (DT) e o Período (T) do processo, que é muito utilizado nos cursos de treinamento por questões práticas (CDTN/CNEN, 1997).

\section{Conclusão}

Este trabalho buscou a melhoria dos processos do reator TRIGA IPR-R1, proporcionando a simulação dos parâmetros neutrônicos, levando em consideração os novos conceitos e sistemas, que são baseados em microprocessadores e utilizam instrumentos virtuais e monitores de vídeo típicos do estado da arte e das atuais salas de controle. O presente estudo segue as recomendações da Agência Internacional de Energia Atômica, que sugere o uso de equipamentos e recursos digitais para melhorar a confiabilidade e aumentar a flexibilidade utilizando interfaces homem-máquina amigáveis (IAEA, 2000, 1999a, 1999b) (IEEE, 2003). 
O sistema desenvolvido poderá ser utilizado no treinamento dos operadores das usinas nucleares de potência como parte de sua formação. Poderá também ser facilmente modificado e ser utilizado como um sistema supervisório para o reator nuclear de pesquisa TRIGA IPR-R1.

\section{Agradecimentos}

Esta pesquisa é apoiada pelo Centro de Desenvolvimento da Tecnologia Nuclear (CDTN), pela Comissão Nacional de Energia Nuclear (CNEN), pela Fundação de Amparo à Pesquisa do Estado de Minas Gerais (FAPEMIG), pelo Conselho Nacional de Desenvolvimento Científico e Tecnológico (CNPq) e pelo Instituto Nacional para Reatores Nucleares Inovadores (INCTRNI).

\section{Referências}

CENTRO DE DESENVOLVIMENTO DA TECNOLOGIA NUCLEAR. Curso de treinamento de operadores em reatores de pesquisa. Belo Horizonte: CDTN, 1997.

CENTRO DE DESENVOLVIMENTO DA TECNOLOGIA NUCLEAR. Reator multipropósito brasileiro-escopo preliminar de projeto. Relatório executivo da Diretoria de Pesquisa e Desenvolvimento. Rio de Janeiro, 2008.

IEEE standard criteria for digital computers in safety systems of nuclear power generating stations. Std. 7-4.3.2-2010. 2010. Disponível em: $<$ http://ieeexplore.ieee.org/document/5542302/references $>$. Acesso em: 26 set. 2016.

INTERNATIONAL ATOMIC ENERGY AGENCY. Operational limits and conditions and operating procedures for research reactors: safety guide. Vienna: IAEA, 2008. (IAEA safety standards series, n. NS-G-4.4).

INTERNATIONAL ATOMIC ENERGY AGENCY. Instrumentation and control systems important to safety in nuclear power plants: safety guide. Vienna: IAEA, 2002. (IAEA safety standards series, n. Ns-G-1.3).

INTERNATIONAL ATOMIC ENERGY AGENCY. Software for computer based systems important to safety in nuclear power plants. Vienna: IAEA, 2000. (IAEA safety standards series, n. NSG-1.1).

INTERNATIONAL ATOMIC ENERGY AGENCY. Modern instrumentation and control for nuclear power plants: a guidebook. Vienna: IAEA, 1999. (IAEA technical reporter n. 387).

INTERNATIONAL ATOMIC ENERGY AGENCY. Specifications of requirements for upgrades using digital instrumentation and control systems. Vienna: IAEA, 1999. (IAEA tecdoc n. 1066).

MEASUREMENT and automation: graphical programming for instrumentation. Austin: National Instrumentss, 2007.

MESQUITA, A. Z. Investigação experimental da distribuição de temperaturas no reator nuclear de pesquisa TRIGA IPR-R1. 2005, 181 f. Tese (Doutorado em Engenharia Química). Universidade Estadual de Campinas, Campinas, 2005.

MESQUITA, A. Z.; SOUZA, R. M. G. P. Thermal-hydraulic and neutronic experimental research in the TRIGA reactor of Brazil. Progress in Nuclear Energy, v. 76, p. 183-190, sept. 2014.

PEDERSON, N. Building a nuclear reactor control system upgrade with NI labview and fieldpoint. Austin: National Instruments. 2005.

PINTO, A .J. Sistema digital para simulação de parâmetros neutrônicos do reator nuclear de pesquisa TRIGA IPR-R1. 2011. 107 f. Dissertação (Mestrado em Ciência e Tecnologia das Radiações, Minerais e Materiais). Centro de Desenvolvimento da Tecnologia Nuclear, Belo Horizonte, 2011.

TIGLIOLE, A. B.; et al. Operational experience with the TRIGA reactor of the University of Pavia. In: WORLD TRIGA USERS CONFERENCE, 4., 2008, Lion, Procededings...Lion, 2008. p. 7-10.

Sobre o autor 